UCT-TP-285/11

April 2011

\title{
Observability of an induced electric dipole moment of the neutron from nonlinear QED
}

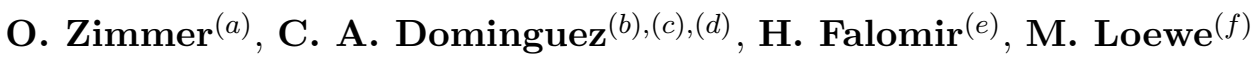 \\ (a) Institut Laue Langevin, 38042 Grenoble, France \\ ${ }^{(b)}$ Centre for Theoretical \& Mathematical Physics,University of Cape Town, Rondebosch 7700, \\ South Africa \\ (c) Department of Physics, Stellenbosch University, Matieland 7600, South Africa \\ (d) National Institute of Theoretical Physics, Private Bag X1, Matieland 7602, South Africa \\ (e) Instituto de Fisica La Plata, Consejo Nacional de Ciencia y Tecnica, Facultad de Ciencias \\ Exactas, Universidad Nacional de La Plata, Argentina \\ ${ }^{(f)}$ Facultad de Física, Pontificia Universidad Católica de Chile, Casilla 306, Santiago 22, Chile
}

\begin{abstract}
It has been shown recently that a neutron placed in an external quasistatic electric field develops an induced electric dipole moment $\mathbf{p}_{\text {IND }}$ due to quantum fluctuations in the QED vacuum. A feasible experiment which could detect such an effect is proposed and described here. It is shown that the peculiar angular dependence of $\mathbf{p}_{\text {IND }}$ on the orientation of the neutron spin leads to a characteristic asymmetry in polarized neutron scattering by heavy nuclei. This asymmetry can be of the order of $10^{-3}$ for neutrons with epithermal energies. For thermalized neutrons from a hot moderator one still expects experimentally accessible values of the order of $10^{-4}$. The contribution of the induced effect to the neutron scattering length is expected to be only one order of magnitude smaller than that due to the neutron polarizability from its quark substructure. The experimental observation of this scattering asymmetry would be the first ever signal of nonlinearity in electrodynamics due to quantum fluctuations in the QED vacuum.
\end{abstract}

PACS numbers: 12.20.Ds, 11.10.-z, 11.10.Lm, 29.27.Hj, 14.20.Dh 


\section{Introduction}

Classical electrodynamics is well known to be a linear theory leading to the superposition principle. At the quantum level the basic QED Lagrangian remains quadratic in the electromagnetic fields, so that the theory still appears to be linear. However, quantum fluctuations in the QED vacuum induce nonlinear effects that lead to a breakdown of the superposition principle [1. In particular, these QED fluctuations make the vacuum appear as an electrically and magnetically polarizable medium. The size of these corrections in nonlinear QED (NLQED) is very tiny, so that experiments with ultra-high intensity lasers have been proposed to search for these effects, e.g. $\mathrm{e}^{+} \mathrm{e}^{-}$pair production from the vacuum [2]-[3], vacuum birefringence [4]-[5], light diffraction by a strong standing electromagnetic wave [6], and nonlinear Compton scattering [7]. A different proposal, involving quasistatic external electromagnetic fields interacting with given electric or magnetic sources, has been made recently [8]-[9]. In [8] general expressions were obtained for the induced electric and magnetic fields in such circumstances, and applied to the case of an electrically charged sphere in the presence of an external, quasistatic magnetic field. As a result of QED nonlinearity there appears an induced magnetic dipole moment, as well as corrections to the Coulomb field of the sphere. In spite of this being a dramatic effect, experimental detection appears very challenging. The complementary case of a purely magnetic dipole moment placed in an external, quasistatic electric field $\mathbf{E}_{0}$ was considered in [9]. The result is an induced electric dipole moment $\mathbf{p}_{\text {IND }}$, plus corrections to the magnetic field produced by the magnetic dipole. It was then suggested that the neutron could be used as a probe in the presence of large electric fields of order $\left|\mathbf{E}_{0}\right| \simeq 10^{10} \mathrm{~V} / \mathrm{m}$, such as present in certain crystals. A distinctive feature of this induced electric dipole moment, which should help in its detection, is its peculiar dependence on the angle between $\mathbf{p}_{\text {IND }}$ and $\mathbf{E}_{0}$, or equivalently the angle between $\mathbf{p}_{\text {IND }}$ and the neutron spin. In this paper we follow up on the experimental observability of such an induced electric dipole moment of the neutron. On the theoretical side we complete the analysis of [9] by computing the interaction Hamiltonian of the neutron immersed in a large external quasistatic electric field $\mathbf{E}_{0}$, and an external, quasistatic, magnetic field $\mathbf{B}_{0}$ of ordinary strength. Given the nonlinearity of the problem one needs to check that (a) the magnetic interaction energy is of the usual form, (nonlinear magnetic corrections due to $\mathbf{B}_{0}$ are negligible), and (b) that the induced electric dipole does interact with the electric field $\mathbf{E}_{0}$ that generates it. The latter interaction energy is expected to have the standard functional form $H_{\text {int }} \propto \mathbf{p}_{\text {IND }} \cdot \mathbf{E}_{0}$, albeit with an a-priori unknown coefficient which we determine. Next, we study the quantum behaviour of $\mathbf{p}_{\text {IND }}$ by means of the Heisenberg equation of motion. This is important for experiments based on potential changes in the Larmor frequency of the neutron spin around an external magnetic field due to the presence of $\mathbf{p}_{\text {IND. }}$. We find no effect here, thus ruling out experiments of this type to detect an induced electric dipole moment of the neutron. Finally, we discuss in some detail a different approach based on neutron-nucleus scattering and conclude that this experiment offers an excellent opportunity to observe such an effect. This is due to the peculiar angular dependence of $\mathbf{p}_{\text {IND }}$. We find that for sufficiently large momentum transfers, a scattering asymmetry is induced with such particular characteristics that it would be easy to distinguish from other standard effects.

The experimental discovery of such an asymmetry would be the first ever signal of a nonlinear effect in electrodynamics due to quantum fluctuations in the QED vacuum. 


\section{Induced electric dipole moment of the neutron}

An appropriate framework to discuss nonlinear effects induced by quantum fluctuations in the QED vacuum is that of the Euler-Heisenberg Lagrangian [1]. This is obtained from the weak field asymptotic expansion of the QED effective action at one loop order leading to

$$
\mathcal{L}_{\mathrm{EH}}^{(1)}=\zeta\left(4 \mathcal{F}^{2}+7 \mathcal{G}^{2}\right)+\ldots,
$$

where the omitted terms are of higher order in the expansion parameter $\zeta$. In SI units

$$
\zeta=\frac{2 \alpha_{\mathrm{EM}}^{2} \epsilon_{0}^{2} \hbar^{3}}{45 m_{e}^{4} c^{5}} \simeq 1.3 \times 10^{-52} \frac{\mathrm{Jm}}{\mathrm{V}^{4}}
$$

with $\alpha_{\mathrm{EM}}=e^{2} /\left(4 \pi \epsilon_{0} \hbar c\right)$ the electromagnetic fine structure constant, $m_{\mathrm{e}}$ and $e$ are the mass and charge of the electron, respectively, and $c$ the speed of light. The invariants $\mathcal{F}$ and $\mathcal{G}$ are defined as

$$
\begin{gathered}
\mathcal{F}=\frac{1}{2}\left(\mathbf{E}^{2}-c^{2} \mathbf{B}^{2}\right)=-\frac{1}{4} F_{\mu \nu} F^{\mu \nu}, \\
\mathcal{G}=c \mathbf{E} \cdot \mathbf{B}=-\frac{1}{4} F_{\mu \nu} \widetilde{F}^{\mu \nu},
\end{gathered}
$$

with $F_{\mu \nu}=\partial_{\mu} A_{\nu}-\partial_{\nu} A_{\mu}$ and $\widetilde{F}^{\mu \nu}=\frac{1}{2} \epsilon^{\mu \nu \rho \sigma} F_{\rho \sigma}$. The so-called critical field $E_{\mathrm{c}}$, which plays the role of a reference field strength for the onset of nonlinearity, is given by

$$
E_{\mathrm{c}}=\frac{m_{\mathrm{e}}^{2} c^{3}}{\hbar e} \simeq 1.3 \times 10^{18} \frac{\mathrm{V}}{\mathrm{m}} .
$$

This estimate is obtained by computing the electric field needed to produce an electron-positron pair in a spatial length of one Compton wavelength. For fields stronger than $E_{\mathrm{c}}$ the weak-field asymptotic expansion leading to Eq. (1) breaks down. In [8] general expressions were obtained for electric and magnetic fields induced by nonlinearity, to leading order in $\zeta$, in the presence of external quasistatic weak fields (smaller than $E_{\mathrm{c}}$ ) and arbitrary sources. These induced fields are

$$
\begin{gathered}
\mathcal{E}(\mathbf{x})=\frac{\zeta}{2 \pi \epsilon_{0}^{2}} \nabla_{\mathbf{x}} \int \frac{\mathrm{d}^{3} y}{|\mathbf{x}-\mathbf{y}|} \nabla_{\mathbf{y}} \cdot\left(4 \mathcal{F}_{\mathrm{M}} \mathbf{D}_{\mathrm{M}}+\frac{7}{c} \mathcal{G}_{\mathrm{M}} \mathbf{H}_{\mathrm{M}}\right), \\
\mathcal{B}(\mathbf{x})=\frac{\zeta}{2 \pi \epsilon_{0}^{2} c^{2}} \nabla_{\mathbf{x}} \times \int \frac{\mathrm{d}^{3} y}{|\mathbf{x}-\mathbf{y}|} \nabla_{\mathbf{y}} \times\left(-4 \mathcal{F}_{\mathrm{M}} \mathbf{H}_{\mathrm{M}}+7 c \mathcal{G}_{\mathrm{M}} \mathbf{D}_{\mathrm{M}}\right),
\end{gathered}
$$

where $\mathbf{D}_{\mathrm{M}}$ and $\mathbf{H}_{\mathrm{M}}$ are the Maxwell (classical) fields produced by the arbitrary sources. Notice that these fields vanish as $\hbar \rightarrow 0(\zeta \rightarrow 0)$. In the case of a current density uniformly distributed on the surface of a sphere of radius $a$, or equivalently, for a uniformly magnetized sphere of the same radius, the Maxwell, magnetic dipole type field, is given by

$$
\mathbf{B}_{\mathrm{d}}=\frac{\mu_{0}}{4 \pi}\left\{\frac{3\left(\mathbf{m} \cdot \mathbf{e}_{r}\right) \mathbf{e}_{r}-\mathbf{m}}{r^{3}} \Theta(r-a)+\frac{2 \mathbf{m}}{a^{3}} \Theta(a-r)\right\}
$$

where $\mathbf{m}$ is identified with the magnetic dipole moment of the source, and $\mathbf{e}_{r}$ is a unit vector in the radial direction. Since the central expressions, Eqs.(6) and (7), were derived assuming $E \equiv c B<E_{\mathrm{c}}$ the following constraint follows

$$
\frac{|\mathbf{m}|}{a^{3}}<\frac{2 \pi m_{\mathrm{e}}^{2} c^{2}}{\hbar e \mu_{0}}
$$


For instance, if $|\mathbf{m}|=0.96 \times 10^{-26} \mathrm{~A} \mathrm{~m}^{2}$, as for the neutron, then it follows that $a \gtrsim 10 \mathrm{fm}$. If this magnetic source is placed in an external, constant electric field $\mathbf{E}_{0}$ it has been shown [9] that there is an induced electric field of the dipole type

$$
\mathcal{E}(\mathbf{x})=-\nabla_{\mathbf{x}}\left[\frac{1}{4 \pi \epsilon_{0}} \frac{\mathbf{p}(\psi)_{\mathrm{IND}} \cdot \mathbf{e}_{r}}{|\mathbf{x}|^{2}}\right]+\mathcal{O}\left(|\mathbf{x}|^{-6}\right) .
$$

where $\psi$ is the angle between the external electric field, lying in the $x-z$ plane, and the magnetic dipole moment pointing along the $z$ axis, i.e. $\mathbf{E}_{0}=\left|\mathbf{E}_{0}\right|\left(\sin \psi \mathbf{e}_{x}+\cos \psi \mathbf{e}_{z}\right)$, and $\mathbf{m}=|\mathbf{m}| \mathbf{e}_{z}$. The induced electric dipole moment $\mathbf{p}(\psi)_{\text {IND }}$ is given by

$$
\mathbf{p}(\psi)_{\mathrm{IND}}=\frac{\zeta \mu_{0}|\mathbf{m}|^{2}\left|\mathbf{E}_{0}\right|}{10 \pi \epsilon_{0} a^{3}}\left[36 \frac{\mathbf{E}_{0}}{\left|\mathbf{E}_{0}\right|}-49\left(\frac{\mathbf{E}_{0}}{\left|\mathbf{E}_{0}\right|} \cdot \mathbf{e}_{x}\right) \mathbf{e}_{x}\right] .
$$

This induced electric field is of the electric dipole type in its radial $1 /|\mathbf{x}|^{3}$ dependence, but it has a manifestly peculiar angular dependence. For instance, along the $z$ axis, and unlike a standard electric dipole field, it has a non-zero component along $\mathbf{e}_{\theta}$ that depends on the azimuthal angle $\phi$. It also has a non-zero component along the direction of $\mathbf{e}_{\phi}$, as may be appreciated by writing the induced electric field in spherical coordinates $(r, \theta, \phi)$, i.e.

$$
\begin{aligned}
\mathcal{E}(\mathbf{x})= & \frac{\zeta \mu_{0}|\mathbf{m}|^{2}\left|\mathbf{E}_{0}\right|}{40 \pi^{2} \epsilon_{0}^{2} a^{3}|\mathbf{x}|^{3}}\left\{2[36 \cos \theta \cos \psi-13 \sin \theta \cos \phi \sin \psi] \mathbf{e}_{r}\right. \\
& \left.+[13 \cos \theta \cos \phi \sin \psi+36 \sin \theta \cos \psi] \mathbf{e}_{\theta}-13 \sin \phi \sin \psi \mathbf{e}_{\phi}\right\} .
\end{aligned}
$$

In addition to the induced electric field Eq.(10), there is an induced magnetic field (a correction to the field produced by the magnetic dipole source), which can be derived from a vector potential, i.e. $\mathcal{B}(\mathbf{x})=\nabla \times \mathcal{A}(\mathbf{x})$, where after a lengthy calculation one finds

$$
\begin{aligned}
\mathcal{A}(\mathbf{x})= & \frac{\zeta \mu_{0}}{4 \pi \epsilon_{0}|\mathbf{x}|^{2}}\left\{4\left|\mathbf{E}_{0}\right|^{2}\left(\mathbf{e}_{r} \times \mathbf{m}\right)-7\left[\mathbf{m} \cdot \mathbf{E}_{0}+3\left(\mathbf{E}_{0} \cdot \mathbf{e}_{r}\right)\left(\mathbf{m} \cdot \mathbf{e}_{r}\right)\right]\left(\mathbf{e}_{r} \times \mathbf{E}_{0}\right)\right. \\
& \left.+7\left(\mathbf{E}_{0} \cdot \mathbf{e}_{r}\right)\left(\mathbf{m} \times \mathbf{E}_{0}\right)\right\}\left[1+\mathcal{O}\left(\frac{\mu_{0}|\mathbf{m}|^{2}}{a^{6} \epsilon_{0}\left|\mathbf{E}_{0}\right|^{2}}\right)\right]+\mathcal{O}\left(|\mathbf{x}|^{-4}\right) .
\end{aligned}
$$

Notice that while $\mathcal{E}$ grows linearly with $\left|\mathbf{E}_{\mathbf{0}}\right|, \mathcal{B}$ depends quadratically on $\left|\mathbf{E}_{\mathbf{0}}\right|$.

We proceed to discuss the interaction energy of the magnetic dipole source and its induced electric dipole with the external constant field $\mathbf{E}_{0}$, and with an external uniform magnetic field $\mathbf{B}_{0}$ weak enough not to induce nonlinear effects, i.e. $c\left|\mathbf{B}_{0}\right| \ll E_{\mathrm{c}}$. Given the nonlinearity of the problem it is important to verify that the magnetic interaction Hamiltonian has the expected form $-\mathbf{m} \cdot \mathbf{B}_{0}$, given the strength of $\mathbf{B}_{0}$. In addition, the electric interaction energy of the induced electric dipole and the external field $\mathbf{E}_{0}$ is a-priori unknown. This need not be exactly of the form

$$
H_{\text {int }}=-\frac{1}{2} \mathbf{p} \cdot \mathbf{E}_{0},
$$

as one would obtain for a linearly polarizable particle immersed in an external electric field, e.g. for a polarizable neutron on account of its quark substructure. In fact, the electric interaction Hamiltonian due to nonlinearity lacks the factor $1 / 2$ as shown next. The canonical energymomentum tensor is defined as

$$
T_{\nu}^{\mu}=\frac{\partial \mathcal{L}_{\text {tot }}}{\partial\left(\partial_{\mu} A_{\alpha}\right)}\left(\partial_{\nu} A_{\alpha}\right)-\mathcal{L}_{\text {tot }} \delta^{\mu}{ }_{\nu}
$$


where the total Lagrangian density is $\mathcal{L}_{\text {tot }}=\mathcal{L}-j_{\mu} A^{\mu}$, with $\mathcal{L}=\epsilon_{0} \mathcal{F}+\mathcal{L}_{\mathrm{EH}}^{(1)}$ and $\mathcal{L}_{\mathrm{EH}}^{(1)}$ given in Eq.(11). This equation can be rewritten as

$$
\begin{aligned}
T^{\mu}{ }_{\nu}= & \left(\frac{\partial \mathcal{L}}{\partial \mathcal{F}} F^{\mu \alpha}+\frac{\partial \mathcal{L}}{\partial \mathcal{G}} \widetilde{F}^{\mu \alpha}\right) F_{\alpha \nu}-\mathcal{L} \delta^{\mu}{ }_{\nu}+(j \cdot A) \delta^{\mu}{ }_{\nu} \\
& +A_{\nu} \partial_{\alpha}\left(\frac{\partial \mathcal{L}}{\partial \mathcal{F}} F^{\mu \alpha}+\frac{\partial \mathcal{L}}{\partial \mathcal{G}} \widetilde{F}^{\mu \alpha}\right)-\partial_{\alpha}\left[\left(\frac{\partial \mathcal{L}}{\partial \mathcal{F}} F^{\mu \alpha}+\frac{\partial \mathcal{L}}{\partial \mathcal{G}} \widetilde{F}^{\mu \alpha}\right) A_{\nu}\right] .
\end{aligned}
$$

Since the last term on the right hand side above is the total divergence of an anti-symmetric tensor, employing the equations of motion

$$
\partial_{\beta}\left(\frac{\partial \mathcal{L}}{\partial \mathcal{F}} F^{\beta \alpha}+\frac{\partial \mathcal{L}}{\partial \mathcal{G}} \widetilde{F}^{\beta \alpha}\right)=j^{\alpha}
$$

one can define another energy-momentum tensor as

$$
\begin{aligned}
\theta^{\mu}{ }_{\nu} & =T^{\mu}{ }_{\nu}+\partial_{\alpha}\left[\left(\frac{\partial \mathcal{L}}{\partial \mathcal{F}} F^{\mu \alpha}+\frac{\partial \mathcal{L}}{\partial \mathcal{G}} \widetilde{F}^{\mu \alpha}\right) A_{\nu}\right] \\
& =\left(\frac{\partial \mathcal{L}}{\partial \mathcal{F}} F^{\mu \alpha}+\frac{\partial \mathcal{L}}{\partial \mathcal{G}} \widetilde{F}^{\mu \alpha}\right) F_{\alpha \nu}-\mathcal{L} \delta^{\mu}{ }_{\nu}+\left(j_{\alpha} A^{\alpha}\right) \delta^{\mu}{ }_{\nu}-j^{\mu} A_{\nu} .
\end{aligned}
$$

Notice that this tensor is symmetric and gauge invariant except for the last two terms. The total energy density of the system is defined as the component $\theta_{0}^{0}$,

$$
\mathcal{H}_{\text {tot }}=\theta_{0}^{0}=\frac{\partial \mathcal{L}}{\partial \mathbf{E}} \cdot \mathbf{E}-\mathcal{L}-\mathbf{j} \cdot \mathbf{A}=\mathbf{D} \cdot \mathbf{E}-\mathcal{L}-\mathbf{j} \cdot \mathbf{A}
$$

In general, for a given configuration of the fields the interaction Hamiltonian is defined as the difference of the total Hamiltonian with and without the sources. In a quantum theory it is defined as the difference of the total Hamiltonian evaluated at the fields in the interaction picture, with and without the external sources. Then, the interaction Hamiltonian $H_{\text {int }}$, i.e. the volume integral of the interaction Hamiltonian density $\mathcal{H}_{\text {int }}$ is

$$
H_{\text {int }}=\int \mathcal{H}_{\text {int }} \mathrm{d}^{3} r=-\int \mathbf{j} \cdot \mathbf{A} \mathrm{d}^{3} r=-\int \mathbf{j} \cdot\left(\mathbf{A}_{0}+\mathcal{A}\right) \mathrm{d}^{3} r
$$

where $\mathbf{A}=\mathbf{A}_{0}+\mathcal{A}$, with $\mathcal{A}$ given in Eq.(13), and $\mathbf{A}_{0}$ is the vector potential associated with $\mathbf{B}_{0}$, i.e. $\mathbf{B}_{0}=\nabla \times \mathbf{A}_{0}$, and $\mathbf{A}_{0}=\frac{1}{2} \mathbf{B}_{0} \times \mathbf{r}$. The current $\mathbf{j}$ corresponding to the magnetized sphere producing the field, Eq.(8), is $\mathbf{j}=\frac{3}{4 \pi a^{3}} \mathbf{m} \times \mathbf{e}_{r} \delta(r-a)$. In Eq.(20) the self energy of the magnetized sphere, independent of the external field, has been omitted. After performing the integration in Eq.(20) one finds

$$
H_{\mathrm{int}}=-\mathbf{m} \cdot \mathbf{B}_{0}-\mathbf{p}(\psi)_{\mathrm{IND}} \cdot \mathbf{E}_{0},
$$

which has the correct magnetic interaction term as in the linear theory. The electric interaction energy is of the expected form, but it involves a coefficient different from the case of linear QED as a result of nonlinearity. In the absence of the external magnetic field $\mathbf{B}_{0}$, and using Eq.(11), the interaction Hamiltonian becomes

$$
H_{\mathrm{int}}=-\frac{\zeta \mu_{0}|\mathbf{m}|^{2}\left|\mathbf{E}_{0}\right|^{2}}{10 \pi \epsilon_{0} a^{3}}\left(36-49 \sin ^{2} \psi\right)=\frac{\zeta \mu_{0}|\mathbf{m}|^{2}\left|\mathbf{E}_{0}\right|^{2}}{10 \pi \epsilon_{0} a^{3}}\left(13-49 \cos ^{2} \psi\right) .
$$

Notice the dependence of $H_{\text {int }}$ on $a^{-3}$. It should be pointed out that in an experimental situation one would typically be interested in a point magnetic dipole. This source would produce very strong fields in its proximity so that the limit $a \rightarrow 0$ would obviously not be allowed. Instead, 
we assume that even in such a case the large distance solution for the fields is well described by the first order approximation to the effective Lagrangian $\mathcal{L}_{\mathrm{EH}}^{(1)}$ in Eq.(1). We also assume that this solution is robust against short distance modifications of the source as long as its symmetry is preserved. In this sense the parameter $a$ is to be considered as a measure of our ignorance about the higher order corrections to this effective Lagrangian, something necessary when dealing with strong fields. The specific value of $a$ will be discussed later in Section 4 . The fact that $H_{\text {int }}$ depends on the orientation of $\mathbf{m}$ with respect to $\mathbf{E}_{0}$ through the angle $\psi$ can be used as a distinctive feature in the design of an experimental asymmetry as described below in Section 5 .

We consider next the quantum behaviour of $\mathbf{p}_{\text {IND }}$ using the Heisenberg equation of motion. To this end we consider a particle with magnetic dipole moment $\mathbf{m}$ related to the spin through the standard relation $\mathbf{m}=g \hbar \mathbf{S}$, where $g$ is the gyromagnetic ratio. Assuming that the dynamics of this particle is described by the Hamiltonian Eq.(21), and given that $\mathbf{p}_{\text {IND }} \propto|\mathbf{m}|^{2}, H_{\text {int }}$ to first order in $\zeta$ contains only quadratic terms in the spin, whose components are the dynamical variables of the problem. The effective Hamiltonian involving these dynamical variables must be symmetrized in order to ensure Hermiticity. Hence, the quadratic terms in the spin entering the Heisenberg equation of motion lead to the commutator

$$
\left[\left\{S_{i}, S_{j}\right\}, S_{k}\right]=i \epsilon_{j k l}\left\{S_{i}, S_{l}\right\}+i \epsilon_{i k l}\left\{S_{j}, S_{l}\right\}
$$

For a spin $1 / 2$ particle, such as the neutron, we have $S_{i}=\frac{1}{2} \sigma_{i}$, and $\left\{S_{i}, S_{l}\right\}=\frac{1}{2} \delta_{i l}$. In this case,

$$
\left[\left\{S_{i}, S_{j}\right\}, S_{k}\right]=i \epsilon_{j k l} \frac{1}{2} \delta_{i l}+i \epsilon_{i k l} \frac{1}{2} \delta_{j l}=\frac{i}{2}\left(\epsilon_{j k i}+\epsilon_{i k j}\right)=0 .
$$

Therefore, $d \mathbf{S} / d t=0$ so that if one is interested in the time evolution of a spin $1 / 2$ particle, and Eq.(21) describes its effective Hamiltonian, we find no contribution from this leading order nonlinear correction. In other words, the precession of the spin is not affected. This is not the case, though, for spin-one particles. This unfortunate feature rules out experiments to detect the induced electric dipole moment of the neutron based on Larmor frequency changes. A different approach involving neutron scattering off nuclei is discussed next.

\section{Neutron-atom scattering amplitude and cross section}

Scattering of slow neutrons by a free atom can be described by a scattering amplitude in the Born-approximation, which in the center of mass system is given by (see e.g. [10])

$$
f(\mathbf{q}, \mathbf{s})=-\frac{M}{2 \pi \hbar^{2}} \int \exp (i \mathbf{q} \cdot \mathbf{r}) H_{\text {int }}(\mathbf{q}, \mathbf{s}) \mathrm{d}^{3} r .
$$

where $M$ is the reduced mass

$$
M=\frac{m_{\mathrm{n}} m_{\mathrm{A}}}{m_{\mathrm{n}}+m_{\mathrm{A}}}
$$

with $m_{\mathrm{n}}$ the neutron mass, and $m_{\mathrm{A}}$ the mass of the atom. The three-momentum transfer $\mathbf{q}$ is $\mathbf{q}=\mathbf{k}-\mathbf{k}^{\prime}$, with $\mathbf{k}$ and $\mathbf{k}^{\prime}$ the neutron wave vectors before and after scattering, respectively, and $\mathbf{s}$ is the neutron spin in units of $\hbar$. The magnitude of $\mathbf{q}$ will be denoted as $|\mathbf{q}| \equiv q$ in the sequel. The total Hamiltonian $H_{\text {int }}$ involves all known interactions between the neutron and the atom, to which we add now the new interaction due to NLQED given in Eq.(22). Correspondingly, the total scattering amplitude can be written as

$$
f(\mathbf{q}, \mathbf{s})=f_{\mathrm{N}}(\mathbf{q}, \mathbf{s})+f_{\mathrm{MAG}}(\mathbf{q}, \mathbf{s})+f_{\mathrm{e}}(\mathbf{q})+f_{\mathrm{POL}}(\mathbf{q})+f_{\mathrm{SO}}(\mathbf{q}, \mathbf{s})+f_{\mathrm{PV}}(\mathbf{q}, \mathbf{s})+f_{\mathrm{IND}}(\mathbf{q}, \mathbf{s}),
$$


where the various contributions are as follows. The term $f_{\mathrm{N}}(\mathbf{q}, \mathbf{s})$ is due to the hadronic interaction of the neutron with the nucleus, and is usually the dominant term. The amplitude $f_{\mathrm{MAG}}(\mathbf{q}, \mathbf{s})$ corresponds to the interaction of the neutron magnetic moment with the atomic magnetic field (for atoms with unpaired electrons). This term can be of a similar size as $f_{\mathrm{N}}(\mathbf{q}, \mathbf{s})$. The next three terms arise from various electromagnetic interactions, i.e. $f_{\mathrm{e}}(\mathbf{q})$ is due to scattering of the neutron charge radius by the electric charges in the atom, $f_{\mathrm{POL}}(\mathbf{q})$ arises from the electric polarizability of the neutron due to its quark substructure, and $f_{\mathrm{SO}}(\mathbf{q}, \mathbf{s})$ corresponds to the spin-orbit interaction of the neutron in the electric field of the nucleus. The term $f_{\mathrm{PV}}(\mathbf{q}, \mathbf{s})$ is a weak interaction, parity-violating amplitude which we list separately from $f_{\mathrm{N}}(\mathbf{q}, \mathbf{s})$ as it has a different dependence on neutron spin. Finally, $f_{\text {IND }}$ is the new component due to the induced electric dipole moment of the neutron, which we wish to isolate experimentally.

The scattering amplitude, Eq.(27), enters the differential cross section for elastic neutron scattering by a single atom in the ground state,

$$
\frac{\mathrm{d} \sigma}{\mathrm{d} \Omega}(\mathbf{q}, \mathbf{P})=\left\langle|f(\mathbf{q}, \mathbf{s})|^{2}\right\rangle
$$

which includes an ensemble average over nuclear and electronic spin degrees of freedom (if present), and the neutron spin. The incident neutrons are characterized by a polarization defined as $\mathbf{P}=2\langle\mathbf{s}\rangle$. In the absence of nuclear and electronic polarization of the atom, the case of interest here, the kinematic scattering variables are $\mathbf{q}$ and $\mathbf{P}$. Experimentally, one determines neutron scattering cross sections using a sample containing a macroscopic number of atoms. Considering a single atomic species, the ensemble average in Eq.(28) still has to account for the isotopic composition and the different states of total spin of a neutron scattering off a nucleus with non-zero spin. For slow neutrons with wavelengths much larger than the nuclear radius $R_{\mathrm{N}}$ the hadronic amplitude $f_{\mathrm{N}}$ is practically independent of $\mathbf{q}$. This is in the absence of nuclear resonances for thermal and epithermal neutrons, i.e. for the energy range of interest here. Scattering thus proceeds in an s-wave and is isotropic in the center of mass system. One defines a neutron scattering length operator as

$$
a_{\mathrm{N}}(\mathbf{s})=-\lim _{q \rightarrow 0} f_{\mathrm{N}}(\mathbf{q}, \mathbf{s})
$$

For a nucleus with spin $\hbar \mathbf{I}$ one has

$$
a_{\mathrm{N}}(\mathbf{s})=\frac{(I+1) a_{+}+I a_{-}}{2 I+1}+\frac{2\left(a_{+}-a_{-}\right)}{2 I+1} \mathbf{s} \cdot \mathbf{I},
$$

where $a_{+}$and $a_{-}$are the eigenvalues of $a_{\mathrm{N}}(\mathbf{s})$ for the two states of total spin $I \pm 1 / 2$ (see e.g. [10]). For a sample with all nuclear species unpolarized, scattering by the $i$ th isotope enters with statistical weight factors $w_{i+}=\left(I_{i}+1\right) /\left(2 I_{i}+1\right)$ and $w_{i-}=I_{i} /\left(2 I_{i}+1\right)$. The leading term in the cross section is then given by

$$
\overline{\left|a_{\mathrm{N}}\right|^{2}}=\sum_{i} c_{i}\left[w_{i+}\left|a_{i+}\right|^{2}+w_{i-}\left|a_{i-}\right|^{2}\right]
$$

where the bar indicates the averaging over isotopes and spin states, and $c_{i}$ stands for the relative abundance of the $i$ th isotope. In next-to-leading order the cross section contains interference terms between small amplitudes like $f_{\text {IND }}$ and a usually dominant coherent nuclear scattering length $\overline{a_{\mathrm{N}}}$, which for unpolarized nuclei is given by

$$
\overline{a_{\mathrm{N}}}=\sum_{i} c_{i}\left[w_{i+} a_{i+}+w_{i-} a_{i-}\right]
$$


Most scattering lengths $\overline{a_{\mathrm{N}}}$ are found to be positive with typical values of a few fm. Neutron optical measurements determine a coherent bound scattering length $\bar{b}$, related to the corresponding scattering length $\bar{a}$ of a free atom through

$$
\bar{b}=\bar{a}\left(1+\frac{m_{\mathrm{n}}}{m_{\mathrm{A}}}\right) .
$$

This relation includes the contributions $-\lim _{q \rightarrow 0} \overline{f_{i}}$ due to all amplitudes $(i=\mathrm{N}$, POL...) appearing in Eq.(27). Lacking sufficiently accurate theoretical predictions for the nuclear part, however, one cannot even extract from $\bar{b}$ the sum of all non-hadronic components, which normally contribute less than $1 \%$. Instead, one needs to perform measurements for $q \neq 0$. Values for $\bar{b}$ and the total neutron scattering cross section of an atom fixed in space, $\sigma_{\mathrm{s}, \mathrm{b}}=4 \pi \bar{b}^{2}$, can be found e.g. in [11]. For later use we quote the values for lead with natural isotopic abundances

$$
\begin{aligned}
\bar{b} & =(9.401 \pm 0.002) \mathrm{fm}, \\
\sigma_{\mathrm{s}, \mathrm{b}} & =(11.187 \pm 0.007) \times 10^{-24} \mathrm{~cm}^{2} .
\end{aligned}
$$

For low neutron energies one also has to take into account interference effects of the neutron waves scattered from different atoms, as e.g. in condensed-matter studies. Classical examples are Bragg scattering by single crystals and measurements of phonon dispersion relations. However, for sufficiently large momentum transfer $\mathbf{q}$ as considered here, interatomic interferences and eventual nuclear spin correlations between different atoms can be neglected. We thus consider the cross section in the center of mass system as given by

$$
\frac{\mathrm{d} \sigma}{\mathrm{d} \Omega} \simeq \overline{\left|a_{\mathrm{N}}\right|^{2}}+\left\langle\left|f_{\mathrm{SO}}(\mathbf{q}, \mathbf{s})\right|^{2}\right\rangle+\ldots-\sum_{j}\left\langle 2 \operatorname{Re}\left[\overline{a_{\mathrm{N}}} f_{j}(\mathbf{q}, \mathbf{s})\right]\right\rangle .
$$

where the dots stand for the remaining contributions of squared amplitudes from Eq.(27), and the sum is over $j=\mathrm{e}, \mathrm{MAG}, \mathrm{POL}, \mathrm{SO}, \mathrm{PV}$ and IND. The formulas to transform this expression to the laboratory reference frame can be found in Ref. [10]. As long as the nucleus is free to recoil, the total cross section does not change and changes in the angular distribution of the scattered neutrons appear only at order $m_{\mathrm{n}} / m_{\mathrm{A}}$. Since we are not interested in angular distributions and will only consider atoms much heavier than the neutron, we may use the scattering cross section as given above in Eq.(35).

\section{Scattering amplitude due to the NLQED induced electric dipole moment}

We now turn to the calculation of the new amplitude $f_{\text {IND }}$ due to the NLQED induced electric dipole moment $\mathbf{p}_{\text {IND }}$ given in Eq.(11). The magnetic moment of the neutron, $\mathbf{m}$, can be written as

$$
\mathbf{m}=\mu_{\mathrm{n}} \sigma,
$$

where

$$
\mu_{\mathrm{n}}=-9.662 \times 10^{-27} \mathrm{~A} \mathrm{~m}^{2},
$$

and $\sigma=2$ s are the Pauli matrices. From Eq. (9) there follows the lower bound

$$
a>7.6 \mathrm{fm} \text {. }
$$




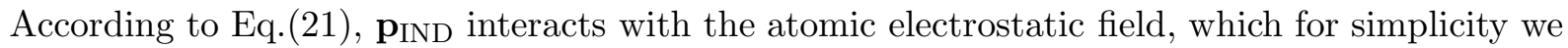
consider as given by a pointlike nucleus with electric charge $Z e$,

$$
\mathbf{E}_{0}=\frac{1}{4 \pi \epsilon_{0}} \frac{Z e}{r^{2}} \mathbf{e}_{r}
$$

As discussed later, one can neglect electric field shielding due to the atomic electrons. Using Eqs.(39), (22) and (25) one obtains

$$
f_{\mathrm{IND}}(q, R, a, \beta)=\frac{\zeta M \mu_{0} \mu_{\mathrm{n}}^{2} Z^{2} e^{2}}{320 \pi^{4} \hbar^{2} \epsilon_{0}^{3} a^{3}}\left[-13 I_{1}(q, R)+49 I_{2}(q, R, \beta)\right],
$$

where $\beta$ is the angle between $\mathbf{s}$ and $\mathbf{q}$. The two integrals $I_{1}(q, R)$ and $I_{2}(q, R, \beta)$ can be easily calculated analytically in polar coordinates with $\mathbf{q}$ along the polar axis. They are given by

$$
I_{1}(q, R)=\int \frac{\exp (i \mathbf{q} \cdot \mathbf{r})}{r^{4}} \mathrm{~d}^{3} r
$$

and

$$
I_{2}(q, R, \beta)=\int \frac{\exp (i \mathbf{q} \cdot \mathbf{r})}{r^{4}}(\cos \beta \cos \theta+\sin \beta \sin \theta \cos \varphi)^{2} \mathrm{~d}^{3} r .
$$

The radial integration extends over all space, excluding a sphere of radius $R$ around the nucleus. For a heavy nucleus like lead, electric fields as strong as $10^{23} \mathrm{Vm}^{-1}$ exist close to the nuclear surface. This exceeds by far the critical field, Eq.(5), beyond which higher order terms in the one-loop effective Lagrangian in Eq.(11) become important [12 and thus cannot be neglected. Therefore, $R$ has to be much larger than the nuclear radius $R_{\mathrm{N}}$, and we choose it here as the distance from the nucleus to where the critical field is reached, i.e.

$$
E_{\mathrm{c}}=\frac{1}{4 \pi \epsilon_{0}} \frac{Z e}{R^{2}}
$$

For lead isotopes with $Z=82$ one has $R \simeq 300 \mathrm{fm}$. The integrals Eqs.(41) and (42) can be solved analytically with the result

$$
\begin{aligned}
I_{1}(q, R) & =\frac{2 \pi}{R}\left\{\cos (q R)+\frac{\sin (q R)}{q R}+\left[\operatorname{Si}(q R)-\frac{\pi}{2}\right] q R\right\} \\
& \simeq \frac{4 \pi}{R}\left[1-\frac{\pi}{4} q R+\frac{1}{6}(q R)^{2}-\ldots\right]
\end{aligned}
$$

where $\operatorname{Si}(x)$ is the Sine integral, and

$$
\begin{aligned}
I_{2}(q, R, \beta)= & \frac{\pi}{4 R}\left\{\frac{1}{(q R)^{2}}\left[2+3(q R)^{2}+\left(6+(q R)^{2}\right) \cos (2 \beta)\right] \cos (q R)\right. \\
& -\frac{1}{(q R)^{3}}\left[2-3(q R)^{2}+\left(6-(q R)^{2}\right) \cos (2 \beta)\right] \sin (q R) \\
& \left.-q R(3+\cos (2 \beta))\left(\frac{\pi}{2}-\operatorname{Si}(q R)\right)\right\} \\
\simeq & \frac{4 \pi}{3 R}\left[1-\frac{3 \pi}{32}(3+\cos (2 \beta)) q R+\frac{1}{10}(2+\cos (2 \beta))(q R)^{2}-\ldots\right] .
\end{aligned}
$$


Using Eqs.(44) and (45) in Eq.(40) one finds the final expression for the scattering amplitude due to the NLQED induced electric dipole moment

$$
\begin{aligned}
f_{\mathrm{IND}}(q, R, a, \beta)= & \frac{\zeta M \mu_{0} \mu_{\mathrm{n}}^{2} Z^{2} e^{2}}{320 \pi^{3} \hbar^{2} \epsilon_{0}^{3} a^{3} R}\left\{\frac{49[1+3 \cos (2 \beta)][q R \cos (q R)-\sin (q R)]}{2(q R)^{3}}\right. \\
& \left.+\frac{1}{4}[43+49 \cos (2 \beta)]\left[\cos (q R)+\frac{\sin (q R)}{q R}+\left(\operatorname{Si}(q R)-\frac{\pi}{2}\right) q R\right]\right\} \\
\simeq & \frac{\zeta M \mu_{0} \mu_{\mathrm{n}}^{2} Z^{2} e^{2}}{24 \pi^{3} \hbar^{2} \epsilon_{0}^{3} a^{3} R}\left\{1-\frac{3 \pi}{320}[43+49 \cos (2 \beta)] q R\right. \\
& \left.+\frac{1}{100}[33+49 \cos (2 \beta)](q R)^{2}-\ldots\right\} .
\end{aligned}
$$

The scattering amplitude $f_{\text {IND }}$ exhibits a welcome peculiar dependence on the angle $\beta$ between the neutron spin $\mathbf{s}$ and the three-momentum transfer $\mathbf{q}$. This dependence introduces an asymmetry which for suitable experimental conditions is essentially free from background contributions due to other well known effects. The largest effect is obtained by evaluating $f_{\text {IND }}$ at $\beta=0$ and at $\beta=\pi / 2$. This feature will then play a crucial role in the experimental detection of the NLQED induced electric dipole moment of the neutron, as discussed in the following section.

\section{Scattering asymmetry due to the NLQED induced electric dipole moment}

We define the scattering asymmetry as

$$
A(q, R, a)=\frac{(\mathrm{d} \sigma / \mathrm{d} \Omega)_{\|}-(\mathrm{d} \sigma / \mathrm{d} \Omega)_{\perp}}{(\mathrm{d} \sigma / \mathrm{d} \Omega)_{\|}+(\mathrm{d} \sigma / \mathrm{d} \Omega)_{\perp}}
$$

where the differential cross section, Eq.(35), is evaluated for two neutron polarization states $\mathbf{P}_{\|}$ and $\mathbf{P}_{\perp}$, parallel and perpendicular to the scattering vector $\mathbf{q}$, respectively. The interference term between the coherent nuclear amplitude and the amplitude of interest leads to

$$
A_{\mathrm{IND}}(q, R, a)=\frac{4 \pi}{\sigma_{\mathrm{s}}} \overline{a_{\mathrm{N}}}\left(f_{\mathrm{IND} \perp}-f_{\mathrm{IND} \|}\right) P
$$

where $f_{\mathrm{IND} \|}=f_{\mathrm{IND}}(q, R, a, 0)$ and $f_{\mathrm{IND} \perp}=f_{\mathrm{IND}}(q, R, a, \pi / 2), P=\left|\mathbf{P}_{\|}\right|=\left|\mathbf{P}_{\perp}\right|$, and $\sigma_{\mathrm{s}}=$ $\sigma_{\mathrm{s}, \mathrm{b}} M^{2} / m_{\mathrm{n}}^{2}$ is the total scattering cross section of the free atom. We argue below that in a welldesigned experiment possible influences of interference terms other than between $\overline{a_{\mathrm{N}}}$ and $f_{\text {IND }}$ are negligible. Hence, $A(q, R, a) \simeq A_{\mathrm{IND}}(q, R, a)$, so that the asymmetry defined in Eq.(47) should allow for a detection and determination of the new amplitude. Using the values for natural lead from Eq.(34) in Eq.(48) one obtains

$$
A(q, R, a) \simeq \frac{f_{\mathrm{IND} \perp}-f_{\mathrm{IND} \|}}{9.5 \mathrm{fm}} P .
$$

From Eqs.(46) and (49) it follows that $A(q, R, a) \propto \chi(q R) / R$, where $\chi$ is a function of the dimensionless parameter $q R$. The maximum of $A(q, R, a)$ occurs for (see Fig. 1)

$$
q R=1.68
$$




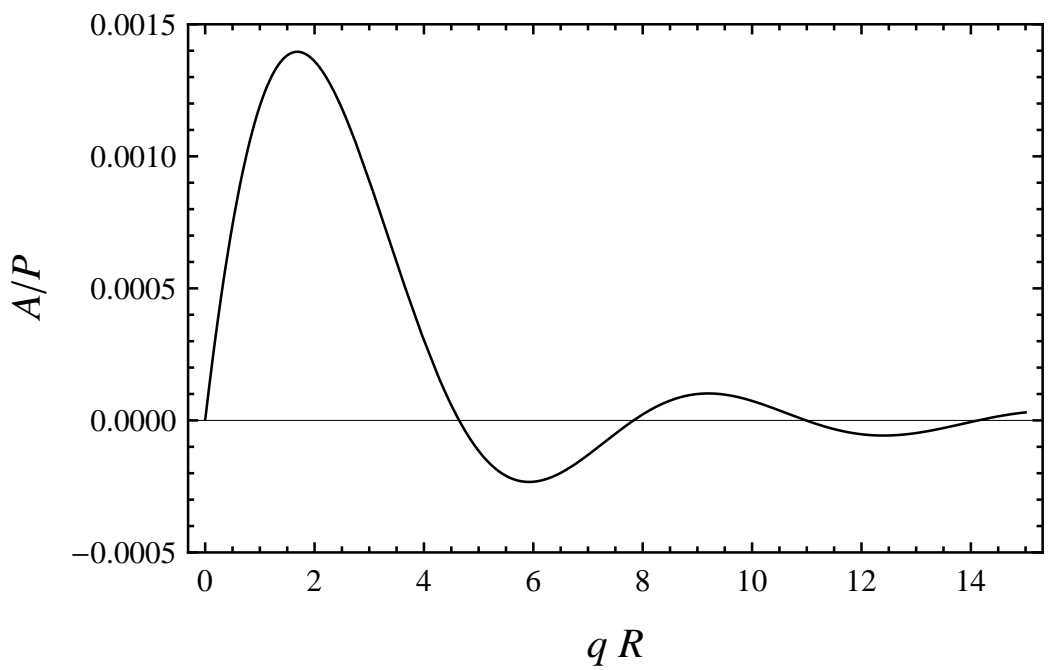

Figure 1: The scattering asymmetry $A(q, R=300 \mathrm{fm}, a=7.6 \mathrm{fm})$, Eq.(49), normalized to the neutron polarization $P$ as a function of the dimensionless variable $q R$.

Using the value of $a$ given in Eq.(38) the asymmetry becomes

$$
A\left(5.6 \times 10^{12} \mathrm{~m}^{-1}, 300 \mathrm{fm}, 7.6 \mathrm{fm}\right)=1.4 \times 10^{-3} P,
$$

a result which appears experimentally accessible. Neglecting nuclear recoil, valid in good approximation for neutron scattering off a heavy target, one may use the relation

$$
q=2 k \sin \frac{\Theta}{2}
$$

where $\Theta$ is the angle between $\mathbf{k}$ and $\mathbf{k}^{\prime}$, and $k \equiv|\mathbf{k}|=2.197 \times 10^{-4} \mathrm{fm}^{-1} \sqrt{E(\mathrm{eV})}$, with $E$ the neutron kinetic energy in $\mathrm{eV}$. In a backscattering geometry, i.e. for $\Theta \simeq \pi$, the maximum asymmetry, Eq.(51), is obtained with epithermal neutrons of energy $E \simeq 165 \mathrm{eV}$. The result for the asymmetry depends explicitly on the radii $R$ and $a$ of spheres centered on the nucleus and on the neutron, respectively. These parameters play the role of separating the short-distance physics close to the electromagnetic sources from the long-distance effects involving fields weak enough for the Euler-Heisenberg approximation to be valid. In this regard it is important to point out that electric and magnetic fields induced by quantum fluctuations in the QED vacuum involve various multipolarities [8]. For instance, in the case of a neutron in an external, quasistatic electric field $\left|\mathbf{E}_{0}\right|<E_{\mathrm{c}}$, the induced electric field Eq.(10) has a dipole type term of order $\mathcal{O}\left(|\mathbf{x}|^{-3}\right)$, as well as a higher multipole of order $\mathcal{O}\left(|\mathbf{x}|^{-6}\right)$, while the induced magnetic field involves terms of order $\mathcal{O}\left(|\mathbf{x}|^{-3}\right), \mathcal{O}\left(|\mathbf{x}|^{-5}\right)$, and $\mathcal{O}\left(|\mathbf{x}|^{-9}\right)$, with $\mathbf{x}=\mathbf{r}-\mathbf{r}_{\mathrm{n}}$. These higher order multipoles can be safely neglected in the interaction energy as long as the weak-field approximation remains valid. However, it is not clear what happens at distances closer to the nucleus or to the neutron. This problem is similar to that of the separation into far and near field regions around a localized charge/current distribution in classical electrodynamics, where the lowest-order multipole provides the long-distance solution. Although one cannot compute the interaction energy due to QED vacuum effects stemming from the regions $r<R$ and $\left|\mathbf{r}-\mathbf{r}_{\mathrm{n}}\right|<a$, their contribution to the asymmetry would presumably have a different dependence on $\mathbf{s}$ and $\mathbf{q}$. For large $q$ it might smear out the oscillations appearing in Fig. 1. For small $q$, corresponding to small spatial resolution in probing the QED vacuum, the asymmetry should not be affected much by the short-distance physics. This statement is underlined by the fact that at leading order in $q R$ the $\beta$-dependent term in $f_{\mathrm{IND}}$, Eq.(46), does not depend on $R$. For $q R \ll 1$, one 
thus obtain a prediction for the asymmetry which should be robust against variations in the choice of $R$, i.e.

$$
A\left(q \ll R^{-1}, R, a\right) \simeq A(q, a)=\frac{49}{320} \frac{\overline{a_{\mathrm{N}}}}{\sigma_{\mathrm{s}}} \frac{\zeta M \mu_{0} \mu_{\mathrm{n}}^{2} Z^{2} e^{2}}{\pi \hbar^{2} \epsilon_{0}^{3} a^{3}} q P
$$

For a neutron energy of $1 \mathrm{eV}$ in a backscattering geometry one expects

$$
A\left(4.4 \times 10^{11} \mathrm{~m}^{-1}, 7.6 \mathrm{fm}\right)=2.2 \times 10^{-4} \mathrm{P} .
$$

From a practical point of view the polarization of epithermal neutrons with more than $100 \mathrm{eV}$ requires a spin filter of polarized protons. This is technically demanding if one wishes to polarize a beam with a diameter of several $\mathrm{cm}$. The measured energy-dependent neutron polarization cross section of a polarized-proton spin filter for the energy range of interest may be found in Ref. [13. On the other hand, neutrons with energies up to $\sim 1 \mathrm{eV}$ are available from a hot moderator in a reactor neutron source with much higher intensity than epithermal neutrons. They can be polarized using magnetic monochromator crystals, or by a spin filter of polarized ${ }^{3} \mathrm{He}$ gas [14] which has a polarization cross section proportional to $k^{-1}$. For the fluxes available at the Institut Laue-Langevin (ILL) in Grenoble an asymmetry as in Eq.(54) appears experimentally accessible within a few days of beam time.

To conclude this section we stress that the neutron scattering asymmetry due to nonlinear QED has two characteristic properties which should make it rather easy to detect and distinguish from other effects. First, the asymmetry attains its maximum value for perpendicular orientations of the neutron polarization and vanishes for opposite orientations. This is in contrast to most ordinary asymmetries which become maximal for opposite orientations. Second, $A_{\text {IND }}(q, R, a)$ exhibits a characteristic $q$ dependence with a broad maximum around the value of $q$ given in Eq.(50). These features are discussed in more detail in the sequel.

\section{Analysis of background asymmetries}

In this section we study the contributions to the asymmetry $A(q, R, a)$, Eq.(47), from the various ordinary scattering amplitudes defined in Eq.(27). The neutron spin-dependent amplitudes can be written as

$$
f(\mathbf{q}, \mathbf{s})=f_{0}(\mathbf{q})+f_{1}(\mathbf{q})[\mathbf{s} \cdot \mathbf{w}(\mathbf{q})]
$$

where $f_{0}(\mathbf{q})$ is spin independent, and $\mathbf{w}$ is a vector not correlated with the neutron spin. In the case of the weak amplitude $f_{\mathrm{PV}}$ the vector $\mathbf{q}$ must be replaced by $\mathbf{k}$. For instance, for the nuclear amplitude in Eq.(30), $\mathbf{w}$ is independent of $\mathbf{q}$ and given by the nuclear spin $\mathbf{I}$. It can be shown in general that the terms proportional to $\left\langle(\mathbf{s} \cdot \mathbf{w})^{2}\right\rangle$ in the differential cross section, Eq.(35), are all independent of the neutron polarization and therefore cannot generate an asymmetry. In principle these terms influence the size of $A(q, R, a)$ through the total scattering cross section $\sigma_{\mathrm{s}}$, Eq.(48). For scattering angles $\Theta \rightarrow 0$ the pure spin-orbit cross section, quadratic in the amplitude $f_{\mathrm{SO}}$, might become large enough to have an impact on $\sigma_{\mathrm{s}}$. However, for sufficiently large $\Theta$, and in the absence of nuclear and electronic polarization, corrections to $\sigma_{\mathrm{s}}$ due to squared-amplitude terms can be safely neglected. The interference terms between the nuclear and the other scattering amplitudes in Eq.(35) may however affect the asymmetry $A(q, R, a)$ through their dependence on the neutron polarization. This requires careful consideration, and we start with the amplitude $f_{\mathrm{SO}}$ for spin-orbit scattering. It originates in the interaction of the neutron magnetic moment with the magnetic field present in the neutron rest frame due to its 
motion through the atomic electric fields. Its expression is (see e.g. [15])

$$
f_{\mathrm{SO}}(\mathbf{q}, \mathbf{s})=i \frac{M}{m_{\mathrm{n}}} \cot (\Theta / 2) \frac{\mu_{\mathrm{n}} \mu_{0}}{2 \pi \hbar} e Z[1-F(q)](\mathbf{s} \cdot \mathbf{n}),
$$

where $e Z[1-F(q)]$ is the Fourier transform of the electric charge density of the atom. This term involves the nuclear charge $Z$ and the atomic form factor $F(q)$ normalized to $F(0)=1$. This form factor is measured e.g. in X-ray scattering off atoms, and is a real function of the momentum. The unit vector $\mathbf{n}$ points along $\mathbf{k} \times \mathbf{k}^{\prime}$, so that the amplitude can contribute only if the neutron polarization has a component out of the scattering plane. The asymmetry due to the spin-orbit interaction is given by

$$
A_{\mathrm{SO}}=\frac{\operatorname{Im} \overline{a_{\mathrm{N}}}}{\sigma_{\mathrm{s}}} \frac{M}{m_{\mathrm{n}}} \cot (\Theta / 2) \frac{\mu_{\mathrm{n}} \mu_{0}}{\hbar} e Z[1-F(q)]\left(\mathbf{P}_{\|} \cdot \mathbf{n}-\mathbf{P}_{\perp} \cdot \mathbf{n}\right) .
$$

The imaginary part of $\overline{a_{\mathrm{N}}}$ above is due to nuclear absorption and can be calculated using the optical theorem. Lead nuclei absorb neutrons only weakly so that $\operatorname{Im} \overline{a_{N}} \simeq(4 \pi)^{-1} k \sigma_{\mathrm{s}}$ for neutron energies of interest here. For a scattering angle $\Theta=\pi / 2$ a neutron kinetic energy of $E \simeq 330 \mathrm{eV}$ would be required to observe the maximum asymmetry according to Eq.(51). In this case the atomic form factor $F(q) \simeq 0$, and a maximum asymmetry $A_{\text {SO }}$ should be observed for $\mathbf{P}_{\perp}$ perpendicular to the scattering plane, i.e. $\mathbf{P}_{\perp} \cdot \mathbf{n}=P$ (while $\mathbf{P}_{\|} \cdot \mathbf{n}=0$ by definition). As a result, for $E=330 \mathrm{eV}$ one has $A_{\mathrm{SO}} \simeq 4.8 \times 10^{-4} P$, while for $E=1 \mathrm{eV}$ together with the conservative value $F(q)=0$, the asymmetry becomes $A_{\mathrm{SO}} \simeq 2.6 \times 10^{-5} P$. These values are already smaller than the corresponding values of $A_{\mathrm{IND}}$, but with suitable experimental settings they can be reduced even further. Notice that the condition $\mathbf{P}_{\perp} \cdot \mathbf{q}=0$ required for $f_{\mathrm{IND} \perp}$ can be realized for different orientations of $\mathbf{P}_{\perp}$, while also fulfilling $\mathbf{P}_{\perp} \cdot \mathbf{n}=0$. Hence, choosing a backscattering geometry, for which $\cot (\Theta / 2) \ll 1$ together with $\mathbf{P}_{\perp} \cdot \mathbf{n} \simeq 0$, and using realistic assumptions about the experimental definition of the directions of $\mathbf{q}$ and $\mathbf{P}$, one can easily suppress $A_{\mathrm{SO}}$ by a factor 50 . Hence, the impact of $A_{\mathrm{SO}}$ can be kept well under the $1 \%$ level.

Next, the amplitude $a_{\mathrm{N}}$ of the neutron-nuclear interaction in Eq.(30), when squared, gives rise to interference between its spin-dependent and spin-independent parts. After ensemble averaging this becomes proportional to $P$ and to the nuclear polarization $P_{\mathrm{N}}$. In thermal equilibrium, $P_{\mathrm{N}}=\tanh \left(\mu_{\mathrm{N}} B /\left(k_{\mathrm{B}} T\right)\right)$ for nuclei with magnetic moment $\mu_{\mathrm{N}}$ in a magnetic field $B\left(k_{\mathrm{B}}\right.$ is the Boltzmann constant). If the target is at room temperature, and given that no magnetic field is needed at the position of the sample, the $P$-dependent cross section is orders of magnitude too small to have an impact on the asymmetry.

We consider next the amplitude $f_{\mathrm{MAG}}$, which is due to the interaction of the neutron magnetic moment with the magnetic field produced by unpaired atomic electrons of paramagnetic contaminants in the sample. The operator structure of this amplitude is given by

$$
f_{\mathrm{MAG}} \propto \mathbf{s} \cdot\left[\mathbf{e}_{q} \times \mathbf{M}(\mathbf{q}) \times \mathbf{e}_{q}\right],
$$

where $\mathbf{M}(\mathbf{q})$ is the Fourier transform of the total (spin and orbital) magnetization of the atom, and $\mathbf{e}_{q}=\mathbf{q} / q$. The interference term with $\overline{a_{\mathrm{N}}}$ thus involves the neutron polarization and the sample-averaged magnetization. It would only influence the asymmetry if (1) the ratio $B / T$ is sufficiently high to result in a sizable magnetization, (2) paramagnetic centers are sufficiently abundant, (3) the two neutron polarization states in the asymmetry have different projections perpendicular to the scattering plane (which is a consequence of the term in brackets in Eq.(58)), and (4) measurements are performed for sufficiently small $q$, where the magnetic form factor still has a sizable value. Regarding the latter, even for the smaller value of $q$ envisaged in Eq.(54), the magnetic form factor leads to a strong suppression of $f_{\mathrm{MAG}}$. Hence, with the conditions 1, 2 and 3 under experimental control one can safely disregard magnetism as a source of an 
asymmetry.

Finally, the parity-violating amplitude $f_{\mathrm{PV}}$ due to the hadronic weak interaction may lead to a different type of asymmetry which has indeed been observed in neutron transmission experiments. Effects depend on the neutron helicity, hence $\mathbf{w}=\mathbf{k}$ in Eq.(55), with a complex coefficient to describe both parity violating spin rotation and transmission asymmetry. The amplitude is normally so small that it requires special efforts to detect it. For thermal neutrons, transmission asymmetries for longitudinally polarized neutrons [16] have typical sizes of a few times $10^{-6}$. However, for neutron energies in the vicinity of p-wave resonances of complex nuclei a strong enhancement due to the weak nuclear interactions may appear. A prominent example is the transmission asymmetry of $7 \%$ found at the p-wave resonance of $0.76 \mathrm{eV}$ in ${ }^{139} \mathrm{La}$ [17]. However, no effect sufficiently strong to affect the asymmetry $A_{\text {IND }}$ is known for lead in the relevant energy range. In addition, an experimental test can easily be performed. In fact, taking the neutron polarization $\mathbf{P}$ parallel and anti-parallel to q, i.e. $\beta=0$ and $\beta=\pi$, it follows from Eqs.(46) and (48) that $A_{\mathrm{IND}}=0$ for these two polarization orientations. In contrast, for $f_{\mathrm{PV}}$ one has $A_{\mathrm{PV}} \propto \sin (\Theta / 2)$, which could be measured separately and corrected for if the need arises.

\section{Comparison of the NLQED amplitude with ordinary electric amplitudes}

The electric amplitudes $f_{\mathrm{POL}}$ and $f_{\mathrm{e}}$ do not generate any known scattering asymmetry. However, owing to their characteristic $q$-dependences a comparison with the NLQED amplitude $f_{\text {IND }}$ is needed. Like $f_{\mathrm{IND}}$, the amplitude $f_{\mathrm{POL}}$ due to the electric polarizability of the neutron, $\alpha_{\mathrm{n}}$, is induced by the nuclear electric field, Eq.(39). In SI units $\alpha_{\mathrm{n}}$ is defined by $\mathbf{p}=4 \pi \epsilon_{0} \alpha_{\mathrm{n}} \mathbf{E}_{0}$, so that its dimension is $\left[\alpha_{\mathrm{n}}\right]=\mathrm{m}^{3}$. The calculation of $f_{\mathrm{POL}}$ follows from Eq.(25) with the interaction energy given by Eq.(14). This leads to the integral Eq.(41) with the result given in Eq.(44). However, the lower limit of the radial integration is now different from that for $f_{\text {IND }}$. In fact, this lower limit can now be extended down to the nuclear radius $R_{\mathrm{N}}$, since for $r>R_{\mathrm{N}}$ the neutron probes only the long-range electric forces. For $r<R_{\mathrm{N}}$ the electric interaction is small in comparison with the nuclear force, so that in early calculations [20]-21] it has simply been included in the nuclear amplitude. In SI units and for the electric field given in Eq.(39), the dependence of $f_{\mathrm{POL}}$ on $q$ is given by

$$
f_{\mathrm{POL}}(q) \simeq \frac{1}{4 \pi \epsilon_{0}} \frac{M}{\hbar^{2}} \frac{Z^{2} e^{2}}{R_{\mathrm{N}}} \alpha_{\mathrm{n}}\left\{1-\frac{\pi}{4} q R_{\mathrm{N}}+\frac{1}{6}\left(q R_{\mathrm{N}}\right)^{2}-\ldots\right\} .
$$

The term linear in $q$ is characteristic of the $r^{-4}$ dependence of the Hamiltonian, and it also enters the interference term in the cross section. This feature has been exploited in the past to measure $\alpha_{\mathrm{n}}$. Conflicting results from experiments performed during more than three decades show that a proper assessment of all systematic errors has been difficult (see e.g. the table in Ref. [22]). The most recent result [23], derived from energy-dependent neutron transmission through a ${ }^{208} \mathrm{~Pb}$ target, and reporting the smallest uncertainty is

$$
\alpha_{\mathrm{n}, \exp }=(1.20 \pm 0.15 \pm 0.20) \times 10^{-3} \mathrm{fm}^{3} .
$$

Calculations using quark bag models [24] agree with this result. An early estimate of Breit and Rustgi [25] using data on pion photoproduction already indicated that $\alpha_{\mathrm{n}}<2 \times 10^{-3} \mathrm{fm}^{3}$. These authors also analyzed other effects which might mimic a signal from the neutron electric polarizability. From an estimate of vacuum polarization effects close to the nucleus, and using the Uehling potential [26, they concluded that this contribution to neutron scattering can be 
safely neglected. Turning now to the question of shielding of the nuclear charge by the atomic electrons, one notices that this might quench the amplitude $f_{\mathrm{IND}}$. Since $f_{\mathrm{POL}}$ and $f_{\mathrm{IND}}$ both depend quadratically on the electric field $\mathbf{E}_{0}$, one can draw parallels with the analysis of $f_{\mathrm{POL}}$. We recall that in our calculation of $f_{\text {IND }}$ one needs to exclude a spherical region of radius $R$ around the nucleus inside which the weak-field expansion of the Euler-Heisenberg Lagrangian breaks down. This procedure was followed (for different reasons) in the early calculations of $f_{\mathrm{POL}}$ [20]-[21], 25] where the nuclear region with radius $R_{\mathrm{N}}$ was excluded. A more recent analysis which does not rely on a simple model for the nuclear charge distribution gives [27]

$$
f_{\mathrm{POL}}(q \rightarrow 0)=\frac{1}{4 \pi \epsilon_{0}} \sqrt{\frac{3}{\pi}} \frac{M}{\hbar^{2}} \frac{Z^{2} e^{2}}{r_{\mathrm{N}}} \alpha_{\mathrm{n}}
$$

where $r_{\mathrm{N}}$ is the root mean square charge radius of the nucleus. This shows that slow neutrons are indeed insensitive to details at this length scale. Notice that this result is nearly identical to the leading term in Eq.(59) after replacing $R_{\mathrm{N}}$ by $r_{\mathrm{N}}$. In the derivation of Eq.(61) the following intermediate result was obtained in [27]

$$
f_{\mathrm{POL}}(q \rightarrow 0) \propto \int_{0}^{\infty}\left|F_{\mathrm{N}}(\kappa)-F(\kappa)\right|^{2} \mathrm{~d} \kappa=\int_{0}^{\infty}\left|F_{\mathrm{N}}(\kappa)\right|^{2} \mathrm{~d} \kappa\left[1-\mathcal{O}\left(R_{\mathrm{N}} / R_{\mathrm{A}}\right)\right],
$$

where $F_{\mathrm{N}}(\kappa)$ and $F(\kappa)$ are the charge form factors of the nucleus and of the electron distribution in the atom, respectively. With $R_{\mathrm{N}} / R_{\mathrm{A}} \simeq 10^{-5}$, shielding of the nuclear charge can be neglected in $f_{\mathrm{POL}}$. Even in the limit $q \rightarrow 0$ the neutron feels the full unscreened nuclear charge as far as the electric polarizability is concerned. For the NLQED induced electric dipole moment it follows that, with $R \simeq 300 \mathrm{fm}$ for lead, the corresponding correction term of order $\mathcal{O}\left(R / R_{\mathrm{A}}\right)$ is much larger than the one of order $\mathcal{O}\left(R_{\mathrm{N}} / R_{\mathrm{A}}\right)$. However, $R / R_{\mathrm{A}} \lesssim 10^{-2}$ is still small enough so that one can neglect shielding of the electric field in the region around the nucleus. We now compare the two amplitudes $f_{\mathrm{POL}}$ and $f_{\mathrm{IND}}$ in the limit $q \rightarrow 0$, i.e. their respective contributions to the neutron scattering length. Setting $R_{\mathrm{N}}=1.2 \mathrm{fm} A^{1 / 3}$ and using Eq.(60) one can estimate the leading order term in Eq.(59) as

$$
f_{\mathrm{POL}}(q \rightarrow 0) \simeq 0.04 \mathrm{fm} .
$$

From Eq.(46) the corresponding leading term in $f_{\text {IND }}$ is

$$
f_{\mathrm{IND}}(q \rightarrow 0)=\frac{1}{4 \pi \epsilon_{0}} \frac{M}{\hbar^{2}} \frac{Z^{2} e^{2}}{R} \alpha_{\mathrm{IND}},
$$

where

$$
\alpha_{\mathrm{IND}}=\frac{\zeta \mu_{0} \mu_{\mathrm{n}}^{2}}{6 \pi^{2} \epsilon_{0}^{2} a^{3}} \simeq \frac{3.3 \mathrm{fm}^{3}}{(a[\mathrm{fm}])^{3}} .
$$

With the value of $a$ from Eq.(9) one obtains

$$
\alpha_{\mathrm{IND}}=7.5 \times 10^{-3} \mathrm{fm},
$$

which is larger than $\alpha_{\mathrm{n}}$, Eq. (60). However, the impact of this polarizability on the amplitude $f_{\text {IND }}$ for $q \rightarrow 0$ is suppressed with respect to $f_{\mathrm{POL}}$ due to $R \gg R_{\mathrm{N}}$. In fact, for lead one obtains

$$
f_{\mathrm{IND}}(q \rightarrow 0) \simeq 0.006 \mathrm{fm} .
$$

This result, though, is not even an order of magnitude smaller than $f_{\mathrm{POL}}(q \rightarrow 0)$, Eq. (63). It would thus contribute about $5 \times 10^{-11} \mathrm{eV}$ to the neutron optical potential of solid lead, which is quite substantial given the high precision of some neutron optical methods. The contribution 


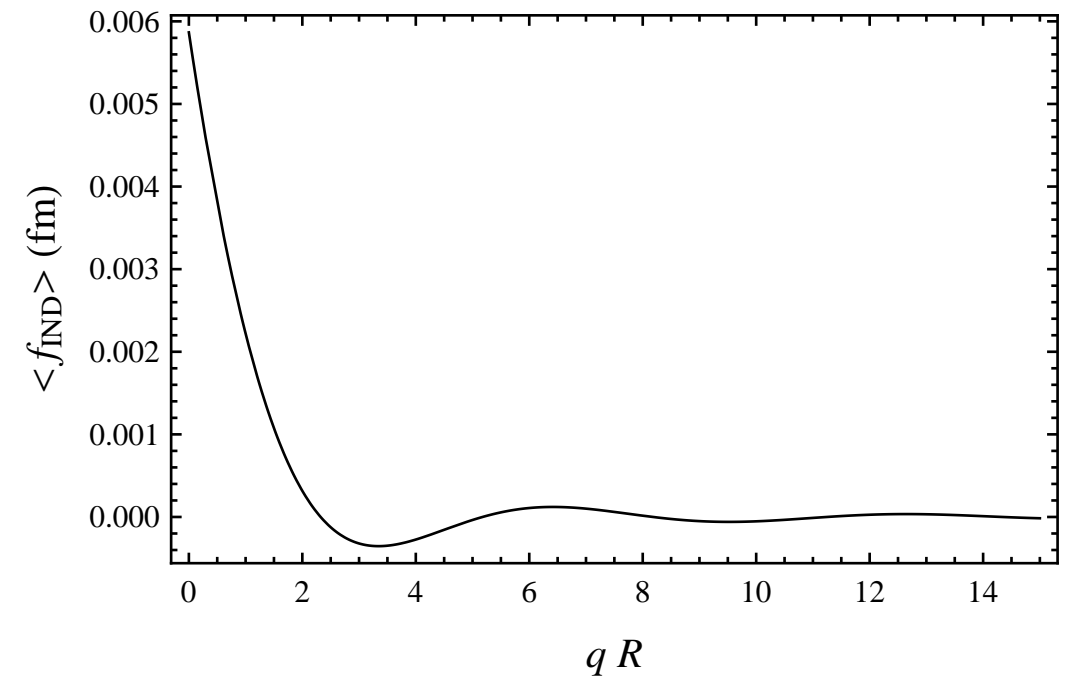

Figure 2: Amplitude $\left\langle f_{\mathrm{IND}}(q, R=300 \mathrm{fm}, a=7.6 \mathrm{fm})\right\rangle$ for unpolarized neutrons, Eq.(69), as a function of the dimensionless variable $q R$.

of the NLQED electric dipole moment to the total cross section is $\sigma_{\mathrm{IND}} \simeq-8 \pi \bar{a} f_{\mathrm{IND}}(q \rightarrow 0)$, where $\overline{a_{\mathrm{N}}} \simeq \bar{a}$ in Eq.(35) has been used. Numerically this becomes

$$
\sigma_{\mathrm{IND}}(q \rightarrow 0)=-0.014 \times 10^{-24} \mathrm{~cm}^{2} .
$$

We discuss next the amplitude $f_{\mathrm{e}}$ which describes the interaction of the electric charges of the atom with the internal charge distribution of the neutron as characterized by its mean squared charge radius. The amplitude for a bound nucleus is given by $f_{\mathrm{e}}=-b_{\mathrm{e}} Z[1-F(q)]$, with the atomic form factor $F(q)$ given in Eqs.(56) and (62) , and the neutron-electron scattering length is $b_{\mathrm{e}} \simeq-1.35 \times 10^{-3} \mathrm{fm}$ as determined from measurements of the total cross sections of lead and bismuth at different neutron energies [18]-[19]. For lead and sufficiently large $q, f_{\mathrm{e}}=0.11 \mathrm{fm}$, which leads to a contribution to the total scattering cross section [27] $\sigma_{\mathrm{e}} \simeq-8 \pi \bar{a} b_{\mathrm{e}} Z \simeq 0.25 \times 10^{-24} \mathrm{~cm}^{2}$. It is also interesting to notice that since $f_{\mathrm{e}}(q \rightarrow 0) \rightarrow 0$ this amplitude does not contribute to the neutron scattering length. The atomic form factor changes significantly at small $q$ where interference of neutron waves from different atoms cannot be neglected. Hence, the macroscopic state of the sample enters crucially in the analysis of scattering data. In contrast, in the case of $f_{\text {IND }}$ where larger effects show up at much higher values of $q$, interatomic interferences do not play any significant role.

To conclude this section we discuss the contribution of $f_{\text {IND }}$ as a potential background in measurements of the amplitudes $f_{\mathrm{POL}}$ and $f_{\mathrm{e}}$ performed with unpolarized neutrons. For the polarization averaged $f_{\text {IND }}$ one obtains

$$
\begin{aligned}
\left\langle f_{\mathrm{IND}}(q, R, a)\right\rangle & =\frac{1}{2} \int_{0}^{\pi} f_{\mathrm{IND}}(q, R, a, \beta) \sin \beta \mathrm{d} \beta \\
& =\frac{\zeta M \mu_{0} \mu_{\mathrm{n}}^{2} Z^{2} e^{2}}{48 \pi^{3} \hbar^{2} \epsilon_{0}^{3} a^{3} R}\left\{\cos (q R)+\frac{\sin (q R)}{q R}+\left[\operatorname{Si}(q R)-\frac{\pi}{2}\right] q R\right\}
\end{aligned}
$$

To analyze the impact on $f_{\mathrm{e}}$ one may approximate relativistic Hartree-Fock results for $F(q)$ by the simple function $\left[1+3\left(q / q_{0}\right)^{2}\right]^{-1 / 2}\left[27\right.$. With $F\left(q_{0}\right)=1 / 2$ the momentum $q_{0}$ provides a scale at which significant changes in $f_{\mathrm{e}}$ take place. For lead, $q_{0}=8.3 \times 10^{10} \mathrm{~m}^{-1}$, and

$$
f_{\mathrm{e}}\left(q_{0}\right)-f_{\mathrm{e}}(0)=50 \times 10^{-3} \mathrm{fm} .
$$


In contrast, the change of $\left\langle f_{\text {IND }}\right\rangle$ is much smaller due to its milder $q$-dependence and its smaller magnitude (see Fig. 2),

$$
\left\langle f_{\mathrm{IND}}\left(q_{0}\right)\right\rangle-\left\langle f_{\mathrm{IND}}(0)\right\rangle=-0.11 \times 10^{-3} \mathrm{fm} .
$$

Since the precision of the best measurements of $b_{\mathrm{e}}$ is at the level of a few percent, potential background due to $\left\langle f_{\text {IND }}\right\rangle$ is negligible. A similar argument leads to the same conclusion for the determination of $\alpha_{\mathrm{n}}$ from $f_{\mathrm{POL}}$.

\section{Conclusions}

Many, if not most proposals to detect nonlinear effects due to quantum fluctuations in the QED vacuum rely on experiments involving lasers of ultra-high intensities [2]-[7]. These intensities, though, are at least two orders of magnitude below current values. An alternative approach has been discussed in this paper, based on the theoretical prediction of an induced electric dipole moment of the neutron, $\mathbf{p}_{\text {IND }}$, in an external quasistatic electric field [9]. The peculiar features of this dipole moment, particularly its dependence on the angle between $\mathbf{p}_{\text {IND }}$ and the neutron spin, suggests the definition of an asymmetry which could be detected in the scattering of polarized neutrons from heavy nuclei. We have introduced this asymmetry and discussed all possible sources of background asymmetries. We have also compared the new NLQED amplitude with ordinary electric scattering amplitudes, particularly the one due to the polarization of the neutron in an electric field due to its quark substructure. The conclusion from this detailed analysis is that the asymmetry due to NLQED should be observable using epithermal neutrons, and even using thermalized neutrons from a hot moderator. This would be the first ever experimental confirmation of nonlinearity in electrodynamics due to QED vacuum fluctuations. The numerical predictions for the asymmetry made in this paper were calculated using definite values for the parameters $R$ and $a$. These were derived from the condition that the electric and magnetic fields should be below their critical values, beyond which the weak-field expansion of the effective Lagrangian breaks down. While the value of the asymmetry $A$ for small $q$ does not depend on $R$, it does depend on $a$ as seen from Eq.(22) Hence, the numerical results given here should be correct up to a numerical factor of order one.

\section{Acknowledgments}

This work was supported in part by FONDECYT 1095217 (Chile), Proyecto Anillos ACT119 (Chile), by CONICET (PIP 01787) (Argentina), ANPCyT (PICT 00909)(Argentina), and UNLP (Proy. 11/X492) (Argentina), NRF (South Africa), and National Institute for Theoretical Physics (South Africa).

\section{References}

[1] W. Heisenberg and H. Euler, Z. Phys. 98, 714 (1936), English translation in W. Korolevski and H. Kleinert, arXiv:physics/0605038; V. Weisskopf, K. Dan. Vidensk. Selsk. Mat. Fys. Medd. 14, 6 (1936), English translation in Early Quantum Electrodynamics: A source book, A.I. Miller ed., (Cambridge University Press, 1994). 
[2] J. Schwinger, Phys. Rev. 82, 664 (1951).

[3] A. Di Piazza, E. Loetstedt, A.I. Milstein, and C.H. Keitel, arXiv:0906.0726.

[4] T. Heinzl, B. Liesfeld, K.U. Amthor, H. Schwoerer, R. Sauerbrey, and A. Wipf, Opt. Commun. 267, 318 (2006).

[5] S.L. Adler, J. Phys. A: Math. Theor. 40, F143 (2007).

[6] A. Di Piazza, K.Z. Hatsagortysan, and C.H. Keitel, Phys. Rev. Lett. 97, 083603 (2008).

[7] Z. Fried, A. Baker, and D. Korff, Phys. Rev. 151, 1040 (1966).

[8] C.A. Dominguez, H. Falomir, M. Ipinza, M. Loewe, and J.C. Rojas, Mod. Phys. Lett. A 24, 1857 (2009).

[9] C.A. Dominguez, H. Falomir, M. Ipinza, S. Kohler, M. Loewe, and J.C. Rojas, Phys. Rev. D 80, 033008 (2009).

[10] V.F. Turchin, Slow neutrons, Israel Program for Scientific Translations Ltd. (1965).

[11] H. Rauch and W. Waschkowski, Neutron scattering lengths, in: Neutron data booklet, Eds. A.-J. Dianoux and G. Lander, Institut Laue Langevin (2002).

[12] G.V. Dunne, in From Fields to Strings, Vol. 1, 445, M. Shifman, A. Vainshtein, and J. Wheater eds. (World Scientific, Singapore, 2005).

[13] V.I. Lushchikov, Yu.V. Taran, and F.L. Shapiro, Sov. J. Nucl. Phys. 10, 669 (1970).

[14] W. Heil, K. Andersen, D. Hofmann, H. Humblot, J. Kulda, E. Lelievre-Berna, O. Schärpf, F. Tasset, Physica B 241-243, 56 (1998).

[15] G.L. Squires, Introduction to the theory of thermal neutron scattering, Cambridge University Press (1978).

[16] M. Forte, B.R. Heckel, N.F. Ramsey, K. Green, G.L. Greene, J. Byrne, J.M. Pendlebury, Phys. Rev. Lett. 45, 564 (1980).

[17] V.P. Alfimenkov, S.B. Borzakov, Vo Van Thuan, et al., Nucl. Phys. A 398, 93 (1983).

[18] L. Koester, W. Nistler, and W. Waschkowski, Phys. Rev. Lett. 36, 1021 (1976).

[19] S. Kopecky, J.A. Harvey, N.W. Hill, M. Krenn, M.Pernicka, P. Riehs, and S. Steiner, Phys. Rev. C 56, 2229 (1997).

[20] V.S. Barashenkov, I.P. Stakhanov, and Yu.A. Aleksandrov, J. Exp. Theor. Phys. 32, 154 (1957).

[21] R.M. Thaler, Phys. Rev. 114, 827 (1959).

[22] J. Schmiedmayer, H. Rauch, and P. Riehs, Nucl. Instr. Meth. A 284, 137 (1989).

[23] J. Schmiedmayer, P. Riehs, J.A. Harvey, and N.W. Hill, Phys. Rev. Lett 66, 1015 (1991).

[24] V. Bernard, B. Hiller, and W. Weise, Phys. Lett. B 205, 16 (1988), and references therein.

[25] G. Breit and M.L. Rustgi, Phys. Rev. 114, 830 (1959).

[26] E.A. Uehling, Phys. Rev. 48, 55 (1935).

[27] V.F. Sears, Phys. Rep. 141, 281 (1986). 